\title{
Avaliação físico-química de amostras de água, sedimento e mata ciliar de uma piscicultura localizada em área agroindustrial à margem do Ribeirão da Mata (MG)
}

\author{
Physicochemical analysis of water, sediment and \\ riparian vegetation of a fish farming located in an agroindustrial area \\ at the border of Ribeirão da Mata (Minas Gerais, Brazil)

\begin{abstract}
Isabela Araujo Torres' ${ }^{1}$ Tarcisio Martins França e Silva ${ }^{2}$, Luciano Santos Rodrigues ${ }^{3}$, Israel Jose Silva ${ }^{4}$, Thais Ataide Costa ${ }^{5}$, Benito Soto-Blanco ${ }^{6}$, Marilia Martins Melo ${ }^{7}$
\end{abstract}

\begin{abstract}
RESUMO
Objetivou-se realizar uma avaliação físico-química de amostras de água, sedimento e mata ciliar em uma piscicultura localizada próxima às margens do Ribeirão da Mata, Minas Gerais. As coletas de água foram semanais em quatro pontos assim denominados: (1) nascente; (2) entrada do sistema; (3) efluente concentrado e (4) ponto de lançamento. Foram monitorados os seguintes parâmetros físico-químicos: temperatura $\left.{ }^{\circ} \mathrm{C}\right)$, $\mathrm{pH}$, oxigênio dissolvido, demanda bioquímica de oxigênio, fósforo total, alcalinidade, sólidos dissolvidos totais, fosfato, nitrato, nitrito, sulfato, amônia e fenol das amostras de água. Foram pesquisados metais pesados (chumbo, cádmio, mercúrio e zinco) e agrotóxicos (organoclorados, organofosforados, clorofosforados, carbamatos, piretroides e rodenticidas anticoagulantes) nas amostras de água, sedimento e mata ciliar. Alcalinidade e sólidos totais apresentaram as maiores concentrações, provavelmente devido às características geológicas,por se tratar de região cárstica. Fenol, em todos os pontos, e fosfato, nos pontos 3 e 4, apresentaram valores acima dos limites permitidos para a classe II de água doce, segundo a Resolução CONAMA no 357. Os outros parâmetros (amônia, nitrato, nitrito e sulfato), emtodos os pontos de coleta, apresentaram baixos valores. Nas amostras de água não foram detectados chumbo e mercúrio; zinco apresentou valores abaixo de 0,007 mg.L.1. As amostras de sedimento apresentaram valores de chumbo entre 11,1566 e 37,0438 mg..-1', estando 12 dessas acima do valor de referência de qualidade. Também em todas as amostras de sedimento foi detectado mercúrio, que variou de 0,35 a 0,82 mg.L.? Em relação ao cádmio, esse não foi detectado nas amostras de sedimento e plantas. Também não foram detectados agrotóxicos em nenhuma das amostras estudadas.
\end{abstract}

Palavras-chave: elementos traço: agrotóxicos; meio ambiente- risco ambiental.

\begin{abstract}
The aim of this study was to carry out a physicochemical analysis of water, sediment and riparian vegetation in a fish farm located near the Ribeirão da Mata basin in Minas Gerais State (Brazil). The water samples were collected weekly at four points: (1) source, (2) system entry, (3) discharge point, (4) and point release. The monitored physicochemical parameters in the water samples were: temperature $\left({ }^{\circ} \mathrm{C}\right), \mathrm{pH}$, dissolved oxygen, biochemical oxygen demand, total phosphorus, alkalinity total dissolved solids, phosphate, nitrate, nitrite, sulfate, ammonia and phenol. Heavy metals (lead, cadmium, mercury and zinc) and pesticides (organochlorine, organophosphates, clorofosforados, carbamates, pyrethroids and anticoagulant rodenticides) were searched in the samples of water, sediment and riparian vegetation. Alkalinity and total solids presented the highest concentration, probably due to geological features of a carstic area. Phenol, at all points, and phosphate, at points 3 and 4, showed values above the limits allowed for class II freshwater, according to the CONAMA Resolution n. 357. The other parameters (ammonia, nitrate, nitrite and sulfate) in all sampling sites showed low values. It was not detected lead and mercury in the water samples; and zinc values were below 0.007 mg.L-1. However, the sediment samples showed lead values from 11.1566 to $37.0438 \mathrm{mg} \cdot \mathrm{L}^{-1}$, with 12 of those samples above the quality reference value. In addition, it was detected mercury in all sediment samples, which ranged from 0.35 to 0.82 mg. L ${ }^{-1}$. It was not detected cadmium in samples of sediment neither plants. No pesticides were detected in the samples.
\end{abstract}

Keywords: trace elements; pesticides; environment; environmental risk.

'Mestranda do curso de pós-graduação em Zootecnia, Escola de Veterinária da Universidade Federal de Minas Gerais (UFMG) - Belo Horizonte (MG), Brasil.

${ }^{2}$ Graduando do curso de Aquacultura da Escola de Veterinária, Laboratório de Saneamento da UFMG - Belo Horizonte (MG), Brasil.

${ }^{3}$ Engenheiro Agrícola, professor adjunto da Escola de Veterinária, Laboratório de Saneamento da UFMG - Belo Horizonte (MG), Brasil.

${ }^{4}$ Médico Veterinário, professor titular da Escola de Veterinária da UFMG - Belo Horizonte (MG), Brasil.

${ }^{5}$ Médica Veterinária, Escola de Veterinária, Laboratório de Toxicologia da UFMG - Belo Horizonte (MG), Brasil.

${ }^{6}$ Médico Veterinário, Professor Associado do Departamento de Clínica e Cirurgia Veterinárias (DCCV). Escola de Veterinária, Laboratório de Toxicologia da UFMG - Belo Horizonte (MG), Brasil. ${ }^{7}$ Médica Veterinária, Professora associada do DCCV da Escola de Veterinária da UFMG - Belo Horizonte (MG), Brasil.

Endereço para correspondência: Marília Martins Melo - Departamento de Clínica e Cirurgia Veterinárias da Escola de Veterinária da UFMG -

Avenida Antônio Carlos, 6.627 - Pampulha - 31270-901 - Belo Horizonte (MG), Brasil. E-mail: mariliamm@ufmg.br

Recebido: 04/02/13 - Aceito: 12/08/16 - Reg. ABES: 110861 


\section{INTRODUÇÃO}

A definição de qualidade da água está diretamente relacionada à sua forma de utilização, e suas características físicas, químicas e biológicas são importantes na avaliação da qualidade ambiental. Por meio do ciclo hidrológico, a água está em permanente contato com os constituintes do meio ambiente (ar e solo), dissolvendo muitos elementos e carreando outros em suspensão (BOM, 2002). Entretanto, a atividade humana vem introduzindo nas águas um número crescente de substâncias.

Um entrave à excelência produtiva do país consiste na questão ambiental, principalmente no tocante à produção em águas doces. Ao longo do tempo, existia - e, em alguns locais, existe até os dias de hoje - a cultura de lançamento de resíduos e dejetos diversos diretamente nos rios, caracterizando uso incorreto e indiscriminado dos recursos naturais, com excessiva ação antrópica sobre eles. Com a magnificação da degradação do meio ambiente, observa-se crescente preocupação dos órgãos regulamentadores com a redução desse fenômeno. A preservação das margens e dos cursos d'água vem ganhando maior impacto, tanto para a própria preservação do meio ambiente como para assegurar os aspectos sociais, econômicos e políticos relacionados.

Diferentes atividades antropogênicas podem liberar substâncias tóxicas para o meio ambiente. Essa liberação pode ser não intencional, como no caso de acidentes e derramamentos; intencional ou deliberada, como pela aplicação de agrotóxicos e pelo lançamento de efluentes industriais sem tratamento (OGA; CAMARGO; BATISTUZZO, 2008). Nesse contexto, os ecossistemas aquáticos estão vulneráveis a diferentes agentes poluidores.

Desde 2000 a.C, vários metais tóxicos têm sido reconhecidos como importantes causas de intoxicações, principalmente entre trabalhadores das áreas de mineração e de refinamento. Fatores como o desenvolvimento industrial, a modernização da malha viária e a mecanização da lavoura foram decisivos para o aumento da poluição ambiental pelos metais tóxicos (MURTA et al., 1997).

O termo metal tóxico se refere a um grupo de elementos que não possui características benéficas nem essenciais aos organismos vivos, produzindo efeitos danosos para as funções metabólicas normais, mesmo em quantidades muito pequenas. Esses constituem a maior fonte poluidora inorgânica de solos e águas, sendo introduzidos no ambiente, principalmente por meio de fertilizantes, pesticidas, combustão de carvão e óleo, emissões veiculares, mineração, fundição, refinamento e incineração de resíduos urbanos e industriais (TAVARES \& CARVALHO, 1992). As plantas podem acumular esses metais em todos os tecidos, podendo transferi-los para a cadeia alimentar, e essa acumulação é um dos temas de interesse ambiental atualmente, pelos efeitos nocivos à saúde animal e humana (MAIGA et al., 2005).

A bacia do Ribeirão da Mata, em Minas Gerais, compreende os municípios de Capim Branco, Confins, Esmeraldas, Lagoa Santa,
Matozinhos, Pedro Leopoldo, Ribeirão das Neves, Santa Luzia, São José da Lapa e Vespasiano. Ela está localizada na margem esquerda do Rio das Velhas. Possui extensão de aproximadamente $817 \mathrm{~km}^{2}$, onde são encontradas riquezas naturais, além de importante patrimônio espeleológico e paleontológico de relevância internacional, localizados na área do Cárste de Lagoa Santa. Especialmente por sua proximidade com o meio urbano e com as atividades industriais, o Ribeirão da Mata recebe diariamente diversos aportes que comprometem sua qualidade (dejetos de indústrias, atividades agropecuárias, esgotos não tratados, entre outros). Outro aspecto importante é o fato de muitos habitantes estarem em contato direto com suas águas e dependerem de seus recursos.

Diante desse contexto, objetivou-se realizar uma avaliação físico-química de amostras de água, sedimento e mata ciliar em uma piscicultura com tanques escavados localizada próxima às margens do Ribeirão da Mata.

\section{METODOLOGIA}

A área de estudo escolhida está situada dentro do município de Pedro Leopoldo, Minas Gerais, que se localiza a $40 \mathrm{~km}$ de Belo Horizonte, a capital do estado, e possui 63.406 habitantes, de acordo com o censo 2014. Dados demonstram que, a maior e a menor atividade econômica no produto interno bruto (PIB) do município pertencem às atividades industriais e agropecuárias, respectivamente (IBGE, 2016).

Este trabalho foi realizado em uma propriedade semiurbana, dotada de aproximadamente 50 tanques escavados, com dimensões variando entre 50 e $200 \mathrm{~m}^{2}$, no período de abril a maio de 2011 . Nos tanques, eram mantidos peixes de várias espécies comerciais como carpa (Cyprinus carpio), surubim (Pseudoplatystoma sp.), matrinxã (Brycon sp.), piau (Leporinus obtusidens), traíra (Hoplias malabaricus) e tilápia (Oreochromis niloticus). O empreendimento está localizado à margem esquerda do Ribeirão da Mata, no município de Pedro Leopoldo, Minas Gerais.

As coletas de água foram semanais em quatro pontos assim denominados:

1. nascente;

2. entrada do sistema;

3. efluente e

4. ponto de lançamento.

Todas as amostras foram coletadas entre 9 e 10 horas.

Para as análises físico-químicas, foram monitorados os seguintes parâmetros: temperatura $\left({ }^{\circ} \mathrm{C}\right), \mathrm{pH}$, oxigênio dissolvido $(\mathrm{OD})$, demanda bioquímica de oxigênio (DBO), fósforo total, alcalinidade e sólidos dissolvidos totais, de acordo com APHA (2005). Para as análises de fosfato, nitrato, nitrito, sulfato, amônia e fenol, utilizou-se método colorimétrico no espectrofotômetro (Micronal, 
modelo B572, São Paulo, SP). Para cada parâmetro, foi utilizado um kit específico (Vacu-vials, CHEMentrics, importado pela Precision Labs, São Paulo, SP), sendo que cada um apresenta um protocolo distinto para a realização de cada análise. As leituras foram feitas com comprimentos de ondas diferentes para cada parâmetro, e os valores fidedignos apresentam uma faixa distinta para cada elemento, tais como: fosfato $\left(4,0\right.$ a 40,0 mg. $\left.\mathrm{L}^{-1}\right)$; sulfato $\left(8,0\right.$ a $\left.100,0 \mathrm{mg} \cdot \mathrm{L}^{-1}\right)$; nitrito $\left(0,08\right.$ a $\left.0,80 \mathrm{mg} . \mathrm{L}^{-1}\right)$; fenol $\left(0,4\right.$ a $\left.8,0 \mathrm{mg} . \mathrm{L}^{-1}\right) ;$ nitrato $(6,0 \mathrm{a}$ 60,0 mg. $\left.\mathrm{L}^{-1}\right)$ e amônia $\left(0,5\right.$ a 7,0 mg. $\left.\mathrm{L}^{-1}\right)$. Ressalta-se que valores acima ou abaixo dessas faixas são estimados pelo espectrofotômetro.

Para determinar a concentração de metais, foram coletadas amostras de água, sedimento e plantas nos mesmos pontos e horários descritos anteriormente. A digestão das amostras para chumbo $(\mathrm{Pb})$, cádmio $(\mathrm{Cd})$, mercúrio $(\mathrm{Hg})$ e zinco $(\mathrm{Zn})$ foram realizadas conforme descrito pela APHA (2005) e a leitura foi feita em espectrofotometria de absorção atômica (SpectrAA-55B- Varian).

Para a pesquisa de agrotóxicos, foram feitos extratos de acordo com a metodologia de Luke, Froberg e Masumoto (1975). Esses foram evaporados completamente e aplicados em placas de sílica (Pre-coated TLC-sheets ALUGRAM Xtra SIL G/Plaques finies CCM) para realização da cromatografia em camada delgada e posterior visualização em radiação ultravioleta, comparados os valores de ration to front (RFs) e revelação com reveladores específicos. Foram pesquisadas as seguintes classes de agrotóxicos: organoclorados (aldrin, alfa $\mathrm{HCH}$, lindano, dieldrin, endrin, hepatacloro, DDT, clordano, mirex e metoxicloro), organofosforados e clorofosforados (clorpirifós, diazinona, metamidofós, mevinfós, acefato, pirimifós, parationa, metidationa e azinfós), carbamatos (carbaril, carbofurano, metomil, propoxur, aldicarbe, oxamil e metiocarbe), piretroides (gama cilatrina, lambda cialotrina, permetrina, deltametrina, ciflutrina, cipermetrina e fenvalerato) e rodenticidas anticoagulantes (brodifacoum e bromadiolone) (LARINI, 1987).

\section{RESULTADOS}

A qualidade da água, sob os aspectos físico-químicos, deve ser considerada em estudos que buscam o entendimento do ecossistema como um todo. Os valores de alcalinidade e sólidos totais (Tabela 1) apresentaram maiores concentrações, que podem ser devido às características geológicas, já que se trata de região cáustica. Ressalta-se que a concentração excessiva de sólidos solúveis suspensos pode causar alterações de cor, sabor, turbidez, além de prejudicar a atividade fotossintética.

A legislação brasileira que trata dos critérios de qualidade da água e padrões de lançamento de efluentes é a Resolução do Conselho Nacional do Meio Ambiente (CONAMA) no 357, de 17/03/2005 (CONAMA, 2005).

Todos os quatro pontos de coleta apresentaram baixos valores para amônia, nitrato, nitrito e sulfato (Tabela 1$)$. Os pontos $3\left(0,7 \mathrm{mg} \cdot \mathrm{L}^{-1}\right)$ e 4 (0,9 mg. $\left.\mathrm{L}^{-1}\right)$ apresentaram fosfato acima do permitido pela resolução do CONAMA.

Em relação aos metais, em todas as amostras de água, os valores de $\mathrm{Pb}$ estavam abaixo de $0,155 \mathrm{mg} \cdot \mathrm{L}^{-1}$. Todavia, foram detectados altos teores de $\mathrm{Pb}$ nas amostras de sedimento que variaram de 11,157 a $37,044 \mathrm{mg} \cdot \mathrm{kg}^{-1}$. E, nas plantas, somente foi detectado $\mathrm{Pb}$ no ponto 1 da segunda coleta $\left(1,702 \mathrm{mg} \cdot \mathrm{kg}^{-1}\right)$ e no ponto 3 da terceira coleta (1,021 mg.kg ${ }^{-1}$ ) (Tabela 2).

Em somente uma amostra de água (ponto 4 da primeira coleta), detectou-se Cd na concentração de $0,029 \mathrm{~mL} . \mathrm{L}^{-1}$. Não foi detectado $\mathrm{Cd}$ nas amostras de sedimento e planta.

Os teores de $\mathrm{Hg}$ no sedimento variaram de $0,35 \mathrm{mg} \cdot \mathrm{kg}^{-1}$ (ponto 1, na primeira coleta) a $0,82 \mathrm{mg} \cdot \mathrm{kg}^{-1}$ (ponto 2 , na terceira coleta). Nas plantas, também foram detectados valores de $0,02 \mathrm{mg} \cdot \mathrm{kg}^{-1}$ (ponto 3 na terceira coleta) a $0,23 \mathrm{mg} \cdot \mathrm{kg}^{-1}$ (ponto 1 na primeira coleta) (Tabela 3 ).

Em relação ao $\mathrm{Zn}$, todas as amostras de água apresentaram valores de $\mathrm{Zn}$ abaixo de $0,007 \mathrm{mg} \cdot \mathrm{kg}^{-1}$. No sedimento, o menor e o maior valor foram observados no ponto 3 , na primeira $\left(18,329 \mathrm{mg} \cdot \mathrm{kg}^{-1}\right)$ e terceira coleta $\left(75,309 \mathrm{mg} \cdot \mathrm{kg}^{-1}\right)$, respectivamente. Já nas plantas, o menor valor de $\mathrm{Zn}$ foi detectado no ponto 3 na quarta coleta $\left(0,752 \mathrm{mg} \cdot \mathrm{kg}^{-1}\right)$ e o maior valor, no ponto 4 na quarta coleta $\left(8,153 \mathrm{mg} \cdot \mathrm{kg}^{-1}\right)$ (Tabela 4$)$.

Tabela 1 - Valores médios de diferentes parâmetros físico-químicos das amostras de água coletadas em quatro momentos, de uma piscicultura localizada em Pedro Leopoldo (MG), em 2011.

\begin{tabular}{|c|c|c|c|c|c|}
\hline \multirow{2}{*}{ Parâmetro } & \multicolumn{4}{|c|}{ Pontos } & \multirow{2}{*}{$\begin{array}{c}\text { VMP } \\
\text { CONAMA }\end{array}$} \\
\hline & 1 & 2 & 3 & 4 & \\
\hline Temperatura $\left({ }^{\circ} \mathrm{C}\right)$ & 21,800 & 22,400 & 22,400 & 22,500 & \\
\hline $\mathrm{pH}$ & 7,200 & 7,200 & 7,400 & 7,400 & \\
\hline Alcalinidade $\left(m g . L^{-1}\right)$ & 203,000 & 201,000 & 185,000 & 188,000 & \\
\hline $\mathrm{OD}\left(\mathrm{mg} \cdot \mathrm{L}^{-1}\right)$ & 5,500 & 6,200 & 6,400 & 6,400 & \\
\hline $\mathrm{DBO}\left(\mathrm{mg} \cdot \mathrm{L}^{-1}\right)$ & 0,970 & 1,280 & 1,440 & 1,540 & \\
\hline $\mathrm{DQO}\left(\mathrm{mg} \cdot \mathrm{L}^{-1}\right)$ & 20,200 & 23,400 & 24,800 & 34,400 & \\
\hline Amônia (mg. $\mathrm{L}^{-1}$ ) & 0,260 & 0,260 & 0,230 & 0,300 & $3,7 \mathrm{mg} \cdot \mathrm{L}^{-1}$ \\
\hline Nitrato (mg. $\left.\mathrm{L}^{-1}\right)$ & 8,200 & 6,700 & 6,400 & 6,000 & $10,0 \mathrm{mg} \cdot \mathrm{L}^{-1}$ \\
\hline Nitrito (mg. $\left.L^{-1}\right)$ & 0,051 & 0,047 & 0,058 & 0,057 & $1,0 \mathrm{mg} \cdot \mathrm{L}^{-1}$ \\
\hline Fosfato (mg...'1) & - & - & 0,700 & 0,900 & 0,3066 mg. $\mathrm{L}^{-1}$ \\
\hline Sulfato (mg. $\mathrm{L}^{-1}$ ) & 8,900 & 9,400 & 8,200 & 8,400 & $250 \mathrm{mg} \cdot \mathrm{L}^{-1}$ \\
\hline Fenol (mg. $\left.\mathrm{L}^{-1}\right)$ & 0,080 & 0,080 & 0,130 & 0,090 & 0,003 mg. $L^{-1}$ \\
\hline $\mathrm{ST}\left(\mathrm{mg} \cdot \mathrm{L}^{-1}\right)$ & 491,000 & 378,000 & 497,000 & 423,000 & \\
\hline STV $\left(m g . L^{-1}\right)$ & 292,000 & 248,000 & 268,000 & 284,000 & \\
\hline STF $\left(m g . L^{-1}\right)$ & 227,000 & 166,000 & 218,000 & 837,000 & \\
\hline
\end{tabular}

VMP CONAMA: valores médios permitidos pelo Conselho Nacional do Meio Ambiente; Pontos: 1: nascente; 2: entrada do sistema; 3: efluente; 4: ponto de lançamento; OD: oxigênio dissolvido; DBO: demanda bioquímica de oxigênio; DQO: demanda química de oxigênio; ST: sólidos totais; STV: sólidos totais voláteis; STF: sólidos totais fixos 
Tabela 2 - Valores de chumbo (mg.kg-1) de amostras de sedimento e plantas coletadas em quatro pontos de uma piscicultura localizada em Pedro Leopoldo (MG), em 2011.

\begin{tabular}{l|c|c|c|c}
$\begin{array}{l}\text { Chumbo } \\
\text { Sedimento }\end{array}$ & $1^{\text {a } \text { coleta }}$ & $2^{\text {a } \text { coleta }}$ & $3^{\text {a coleta }}$ & $4^{\text {a coleta }}$ \\
\hline Ponto 1 & 11,491 & 22,347 & 21,846 & 14,497 \\
\hline Ponto 2 & 30,864 & 23,182 & 37,044 & 29,194 \\
\hline Ponto 3 & 11,157 & 22,347 & 23,683 & 23,182 \\
\hline Ponto 4 & 17,503 & 12,994 & 17,169 & 19,674 \\
\hline Plantas & & & & \\
\hline Ponto 1 & $<0,775$ & 1,7021 & $<0,775$ & $<0,775$ \\
\hline Ponto 2 & $<0,775$ & $<0,775$ & $<0,775$ & $<0,775$ \\
\hline Ponto 3 & $<0,775$ & $<0,775$ & 1,021 & $<0,775$ \\
\hline Ponto 4 & $<0,775$ & $<0,775$ & $<0,775$ & $<0,775$ \\
\hline
\end{tabular}

1: nascente; 2: entrada do sistema; 3: efluente; 4: ponto de lançamento.

Tabela 3 - Valores de mercúrio (mg.kg-1) de amostras de sedimento coletadas em quatro pontos de uma piscicultura localizada em Pedro Leopoldo (MG), em 2011.

\begin{tabular}{l|c|c|c|c}
$\begin{array}{l}\text { Mercúrio } \\
\text { Sedimento }\end{array}$ & $1^{\text {a } \text { coleta }}$ & $2^{\text {a } \text { coleta }}$ & $3^{\text {a coleta }}$ & $4^{\text {a coleta }}$ \\
\hline Ponto 1 & 0,35 & 0,59 & 0,58 & 0,67 \\
\hline Ponto 2 & 0,55 & 0,68 & 0,82 & 0,52 \\
\hline Ponto 3 & 0,63 & 0,49 & 0,53 & 0,52 \\
\hline Ponto 4 & 0,43 & 0,54 & 0,38 & 0,53 \\
\hline Plantas & & & & \\
\hline Ponto 1 & 0,23 & 0,19 & 0,07 & 0,03 \\
\hline Ponto 2 & 0,16 & 0,20 & 0,05 & 0,03 \\
\hline Ponto 3 & 0,02 & 0,23 & 0,07 & 0,17 \\
\hline Ponto 4 & 0,15 & 0,04 & 0,11 & 0,19 \\
\hline
\end{tabular}

1: nascente; 2: entrada do sistema; 3: efluente; 4: ponto de lançamento.

Tabela 4 - Valores de zinco (mg.kg-1) de amostras de sedimento e plantas coletadas em quatro pontos de uma piscicultura localizada em Pedro Leopoldo (MG), em 2011.

\begin{tabular}{l|c|c|c|c}
$\begin{array}{l}\text { Zinco } \\
\text { Sedimento }\end{array}$ & $1^{\text {a coleta }}$ & $2^{\text {a } \text { coleta }}$ & $3^{\text {a coleta }}$ & $4^{\text {a coleta }}$ \\
\hline Ponto 1 & 35,541 & 36,728 & 36,254 & 25,095 \\
\hline Ponto 2 & 46,819 & 33,523 & 45,513 & 51,804 \\
\hline Ponto 3 & 18,329 & 33,167 & 75,309 & 74,478 \\
\hline Ponto 4 & 34,117 & 36,728 & 66,168 & 59,283 \\
\hline Plantas & & & & \\
\hline Ponto 1 & 3,336 & 5,804 & 1,927 & 4,041 \\
\hline Ponto 2 & 2,514 & 3,454 & 2,397 & 5,334 \\
\hline Ponto 3 & 6,626 & 1,809 & 2,749 & 0,752 \\
\hline Ponto 4 & 4,159 & 1,809 & 2,044 & 8,153 \\
\hline
\end{tabular}

1: nascente; 2: entrada do sistema; 3: efluente; 4: ponto de lançamento.

\section{DISCUSSÃO}

Embora seja desconhecida a relação biomassa/volume de água no sistema, quanto maior essa relação, mais amplas e frequentes serão as variações do $\mathrm{pH}$ e da quantidade de OD no meio, determinando condições desfavoráveis à saúde dos organismos cultivados. Observa-se que uma lagoa formada a partir do barramento do curso da nascente (ponto 1), antes da entrada dos tanques e da manutenção de níveis adequados de fitoplâncton (desejável no manejo de tanques de cultivo), contribuiu para a não alteração significativa dos parâmetros $\mathrm{pH}, \mathrm{OD}$, DBO e demanda química de oxigênio (DQO), mantendo a caracterização da qualidade da água dos tanques, bem como a do efluente, dentro dos limites aceitáveis de acordo com a Resolução CONAMA no 357/05 (CONAMA, 2005).

Os resultados obtidos nas análises químicas da água mostraram valores dentro daqueles aceitáveis pela Resolução n 357/05 (CONAMA, 2005) para a classe II de águas doces, com exceção do fenol, que apresentou valor mínimo de $0,08 \mathrm{mg} . \mathrm{L}^{-1}$ e maior de $0,13 \mathrm{mg} . \mathrm{L}^{-1}$; e do fosfato, nas amostras de água dos pontos 3 (efluente concentrado) e 4 (ponto de lançamento), que também ficou acima do limite permitido (Tabela 1). Os outros parâmetros estão dentro do limite máximo.

Os peixes representam o último nível trófico da cadeia alimentar aquática e reagem sensivelmente às alterações ambientais. Tišler $\mathrm{e}$ Zagorc-Končan (1997) compararam a toxicidade do fenol em diferentes organismos aquáticos e constataram que o peixe (Oncorhynchus mykiss) é mais sensível ao fenol do que a alga (Scenedesmus quadricauda) e o crustáceo (Daphnia pulex). De acordo com Pietro et al. (2002), o fenol é extremamente tóxico ao homem, tanto por ingestão como por inalação ou até mesmo por contato dérmico, ainda que em baixas concentrações $\left(1,0 \mathrm{mg} \cdot \mathrm{L}^{-1}\right)$. O fenol aparece nos corpos d'água por meio das descargas de efluentes industriais e domésticos, bem como da hidrólise e da oxidação fotoquímica dos agrotóxicos organofosforados e da degradação microbiana de herbicidas. Alguns compostos fenólicos são resistentes à degradação microbiológica e são transportados a longas distâncias pela água (BOLAÑOS et al., 2001). Sabe-se que a região da piscicultura estudada possui altitude menor (762,402 $\mathrm{m}$ - nascente) do que o nível da rodovia MG-424 ( $800 \mathrm{~m})$ e está aproximadamente a $200 \mathrm{~m}$ de distância da mesma. Também há presença de indústrias, que ficam a menos de $500 \mathrm{~m}$ de distância da nascente que abastece a piscicultura, além de residências próximas à área estudada, podendo ocorrer lixiviação, trazendo compostos tóxicos até os corpos d'água.

Os íons fosfato são despejados no ambiente aquático por meio da lixiviação de rochas, bem como por atividades agrícola e industrial e usos domésticos. Esses são geralmente encontrados em efluentes com baixas concentrações na forma de fosfato orgânico, fosfato inorgânico, ortofosfato e polifosfato (DAS et al., 2006). Na maioria dos ecossistemas, o fósforo é um elemento essencial para crescimento de organismos. No entanto, seu excesso leva à eutrofização dos corpos d'água, 
ocasionando um aumento da população de plantas aquáticas. Em termos ecológicos, esse crescimento na população de plantas aquáticas, especialmente algas, leva a um desbalanceamento na cadeia alimentar do ecossistema, acompanhado de uma diminuição do OD na água. Como consequência, ocorre a mortandade de peixes e crustáceos (MULINARI et al., 2007).

Avaliação de parâmetros como concentração de nitrogenados e sulfatos podem auxiliar no diagnóstico de contaminações por praguicidas e/ou compostos químicos que contenham tais elementos na formulação. Além disso, esses elementos podem ser resultados de descargas industriais e, ao permanecerem na água, podem atingir os organismos aquáticos.No presente estudo, os valores de amônia, nitrato, nitrito e sulfatos ficaram em conformidade com os limites exigidos.

A presença de derivados do nitrogênio na água indica processos biológicos ativos influenciados por poluição orgânica, podendo, em altas concentrações, causar danos fisiológicos e até mortalidade em organismos aquáticos. A amônia difunde-se facilmente por meio das membranas respiratórias, o que causa danos ao epitélio branquial e, como consequência, dificulta as trocas gasosas entre o animal e a água, desestabilizando o sistema de osmorregulação (COSTA et al., 2004).

O nitrito em altas concentrações provoca a oxidação do átomo de ferro da molécula da hemoglobina do sangue, convertendo-a em metahemoglobina, molécula incapaz de transportar oxigênio, estabelecendo-se um quadro de hipóxia e cianose. Diferentemente do que ocorre em ambientes naturais, em que o nitrogênio advém da chuva, do material orgânico e inorgânico de origem alóctone e da fixação de nitrogênio molecular dentro do próprio lago, o principal fator responsável pela presença de amônia nos sistemas de criação de peixes é a entrada de grandes quantidades de compostos orgânicos e inorgânicos, por meio de adubos, fertilizantes e rações, os quais contêm níveis elevados de nitrogênio e fósforo (DUBOROW; CROSBY; BRUNSON, 1997). Foi estudado o metabolismo do matrinxā de água doce, Brycon amazonicus, expostos a $0,6 \mathrm{mg} . \mathrm{L}^{-1}$ de nitrito por $96 \mathrm{~h}$. Foram pesquisadas as concentrações de glicogênio, glicose, lactato, piruvato e amônia, e as atividades das enzimas lactato, glutamato e maleato desidrogenase. A exposição resultou em alteração do perfil metabólico. O catabolismo dos aminoácidos foi exacerbado, agravado por lesão branquial e consequente diminuição da excreção de nitrogênio. Além disso, as enzimas estudadas foram pouco afetadas pela exposição ao nitrito (AVILEZ et al., 2013).

$\mathrm{O}$ sulfato (ânion $\mathrm{SO}_{4}^{-2}$ ) é um dos mais abundantes íons na natureza. Surge nas águas subterrâneas por meio da dissolução de solos e rochas, como o gesso $\left(\mathrm{CaSO}_{4}\right)$ e o sulfato de magnésio $\left(\mathrm{MgSO}_{4}\right)$ e pela oxidação de sulfeto. Nas águas superficiais, ocorre por meio das descargas de esgotos domésticos (por degradação de proteínas) e efluentes industriais.
O valor de referência de qualidade (VRQ) é a concentração de determinada substância no solo ou na água subterrânea, que define um solo como limpo, ou a qualidade natural da água subterrânea, e é determinado com base em interpretação estatística de análises físico-químicas de amostras de diversos tipos de solos e amostras de águas subterrâneas de diversos aquíferos. O valor de prevenção (VP) é a concentração de determinada substância, acima da qual podem ocorrer alterações prejudiciais à qualidade do solo e da água subterrânea. Esse valor indica a qualidade de um solo capaz de sustentar as suas funções primárias, protegendo-se os receptores ecológicos e a qualidade das águas subterrâneas. O valor de intervenção (VI) é a concentração de determinada substância no solo ou na água subterrânea acima da qual existem riscos potenciais, diretos ou indiretos, à saúde humana, considerado um cenário de exposição genérico. Para o solo, ele foi calculado utilizando procedimento de avaliação de risco à saúde humana para cenários de exposição agrícola — área de proteção máxima (APMax) —, residencial e industrial (CETESB, 2005).

O valor de $\mathrm{Pb}$ permitido de VRQ é de $17 \mathrm{~mL} . \mathrm{L}^{-1}$; de VP é $72 \mathrm{~mL} \cdot \mathrm{L}^{-1}$ e o máximo para solo agrícola é de $180 \mathrm{~mL}^{-1}{ }^{-1}$ (CETESB, 2005). Doze amostras de sedimento apresentaram valores de $\mathrm{Pb}$ acima do VRQ, mas ainda abaixo do VP, conforme recomendação da CETESB.

$\mathrm{O} \mathrm{Pb}$ é o metal tóxico presente em maior quantidade na crosta terrestre, sendo de uso industrial bastante difundido (PAOLIELLO \& CHASIN, 2001). Nos últimos anos, a demanda desse metal tem sofrido uma mudança quanto ao tipo de utilização. A contaminação da água pelo $\mathrm{Pb}$, em áreas urbanas e suburbanas, tem sido objeto de estudo de inúmeros pesquisadores. A contaminação do solo pode advir de forma natural ou geológica, ou pode ainda decorrer das atividades exercidas pelo homem, como mineração, indústria e transporte. $\mathrm{O}$ teor de $\mathrm{Pb}$ nos solos varia conforme a região; em regiões próximas de vias de tráfego intenso, os teores são mais elevados do que em áreas isoladas (HENDERSON; BLOOD; RADOSTITS, 1983).

Rosa (2008) pesquisou os teores de metais na água da Bacia Hidrográfica do Rio Mogi-Guaçu, na região de Araras, São Paulo, e relatou o excesso do elemento $\mathrm{Pb}$ devido à contaminação préexistente na bacia.

O valor de Cd permitido de VRQ é de $<0,5 \mathrm{~mL} . \mathrm{L}^{-1}$; de VP é de 1,3 mL.L $\mathrm{L}^{-1}$ e de VI para solo agrícola é de 3,0 mL.L-1 . Todas as amostras de sedimento dessa piscicultura se enquadraram dentro do VRQ, classificando o solo como limpo para $\mathrm{Cd}$.

O Cd é um dos cinco metais mais tóxicos presentes na natureza, não sendo elemento essencial ao homem nem aos animais domésticos. Suas numerosas aplicações industriais e sua capacidade de combinação com outros elementos metálicos (zinco e cobre, principalmente) favorecem a ocorrência de contaminação ambiental (USEPA, 2001). O Cd encontra-se extensamente distribuído no ambiente. É constituinte de produtos como plásticos, pigmentos e baterias, provocando lesões e distúrbios, por meio da contaminação da água, do ar e dos alimentos ingeridos. As principais fontes antropogênicas de Cd são: mineração 
de metais não ferrosos e fundições de metal, combustão de carvão e fabricação de fertilizantes fosfatados. Outras fontes importantes de Cd na hidrosfera incluem águas residuais domésticas, fundição e refino de metais não ferrosos e a fabricação de produtos químicos e metais. Concentrações de Cd de até $5 \mathrm{mg} . \mathrm{kg}^{-1}$ foram relatadas nos sedimentos do rio e lagos, e de 0,03 a 1,00 mg. $\mathrm{kg}^{-1}$ nos sedimentos marinhos (PACYNA \& PACYNA, 2001; OSPAR COMMISSION, 2002).

$\mathrm{O}$ valor de Hg permitido de VRQ é de $0,05 \mathrm{~mL} . \mathrm{L}^{-1}$; de VP é 0,5 mL.L $\mathrm{L}^{-1}$ e de VI para solo agrícola é 12,0 mL.L ${ }^{-1}$ (CETESB, 2005). Todas as 16 amostras de sedimento apresentaram valores de $\mathrm{Hg}$ acima do recomendado de VQR, e 12 ultrapassaram os limites de VP conforme a CETESB (2005). O Hg é um metal utilizado na agricultura, principalmente como fungicida, na amalgamação do ouro e em setores industriais, como na produção de cloro, soda, lâmpadas fluorescentes e termômetros. Dentre os efluentes químicos, o $\mathrm{Hg}$ assume grande relevância para a saúde pública, devido ao fato de ser um metal pesado de efeito cumulativo para o homem, sendo geralmente eliminado nas águas pelos efluentes industriais (GERMANO \& GERMANO, 2001).

O valor de Zn permitido de VRQ é de $60 \mathrm{~mL} . \mathrm{L}^{-1}$; de VP é de $300 \mathrm{~mL} \cdot \mathrm{L}^{-1}$ e de VI para solo agrícola é de 400 mL.L-1 (CETESB, 2005). Três amostras de sedimento apresentaram valores de $\mathrm{Zn}$ acima do recomendado para VRQ. O Zn é um microelemento essencial ou elemento-traço, que exerce funções essenciais ao organismo. Porém, sabe-se que também é tóxico para os peixes, causando mortes, baixo crescimento, alterações nos tecidos, mudança no sistema cardíaco e respiratório, inibindo a desova (SORENSEN, 1991).

Os dados sobre metais pesados no solo ocasionalmente exibem complexos padrões e variações espaciais, possivelmente complicando os esforços para identificar as características desses metais, particularmente em áreas urbanas e industriais. Tais variações e padrões complexos são atribuídos a fenômenos naturais e atividades humanas (antropogênicas) (LIN, 2008). Atividades antropogênicas, como descarga de resíduos do processamento de metais, queima de combustíveis fósseis e uso de pesticidas, causam poluição do solo. Algumas dessas práticas antropogênicas ajudam a distinguir quais os problemas que estão ocorrendo e que podem ser tratados nas decisões políticas relacionadas à poluição ambiental, à avaliação de risco e à remediação (YU-PIN et al., 2010. Como exemplos de problemas, pode-se ressaltar que, na região de estudo, bem perto da piscicultura, existe uma indústria de reciclagem de sucatas, uma indústria de cimento e uma rodovia.

A piscicultura em estudo mostrou índices elevados de $\mathrm{Pb}, \mathrm{Hg}$ e $\mathrm{Zn}$ nas amostras de sedimento. Com esses resultados, podemos presumir que o meio ambiente local está sendo afetado, com risco de contaminação para os peixes, já que os metais pesados são cumulativos.

Nas amostras de água, somente foi detectado Cd em uma amostra, na concentração de $0,029 \mathrm{~mL} \cdot \mathrm{L}^{-1}$. Para água subterrânea, o VI é de $0,005 \mathrm{~mL} . \mathrm{L}^{-1}$, portanto, o valor encontrado pode gerar riscos potenciais, diretos ou indiretos, à saúde humana. Em águas superficiais e subterrâneas, o cádmio pode existir como íons livres, ou como complexos iônicos com outras substâncias orgânicas ou inorgânicas. Compostos solúveis podem migrar na água, sendo o cádmio relativamente imóvel em complexos insolúveis ou adsorvidos em sedimentos. No mar, as formas mais comuns são complexos de íons de cloro e, na água doce, os íons hidratados livres ou gaseificados (dependendo do $\mathrm{pH}$ ) são as formas mais frequentes (EFSA, 2009). Em relação aos sistemas aquáticos, os rios transportam grandes quantidades de cádmio provenientes de intemperismo e de processos de erosão para os oceanos do mundo, tendo sido estimada uma entrada bruta anual de cerca de 15 mil toneladas de cádmio. Além disso, entre 900 e 3.600 toneladas de cádmio são depositadas em ambientes aquáticos em todo o mundo por deposição atmosférica de emissões provenientes de fontes naturais e antropogênicas (UNEP, 2008).

Nas amostras de plantas, em somente uma foi detectado $\mathrm{Pb}$ na concentração de 1,0213 mg.kg-1 no ponto 3 (efluente concentrado). Em relação ao Cd, nas plantas, os valores ficaram menores que $135 \mathrm{mg} \cdot \mathrm{kg}^{-1}$. Em geral, o Cd se acumula nas folhas das plantas e, portanto, é um risco maior para legumes cultivados em solo contaminado do que para sementes ou tubérculos (ALLOWAY; JACKSON; MORGAN, 1990). O tipo de solo também afeta a absorção de Cd pelas plantas. Para solos com o mesmo teor de Cd total, o Cd foi mais solúvel e mais disponível em solos arenosos do que em solos argilosos. E, em todas as amostras de plantas, foi detectado $\mathrm{Hg}$ (que variou de 0,020 a 0,23 mg.kg ${ }^{-1}$ ).

A concentração de metais pesados no meio ambiente, com sua disseminação no solo, na água e na atmosfera tem sido uma crescente preocupação no mundo. Os metais pesados incorporados no solo podem seguir diferentes vias de fixação, liberação e transporte, ficando retidos no solo, seja dissolvidos em soluções ou fixados por processos de adsorção, complexação e precipitação. Também podem ser absorvidos por plantas e incorporados nas cadeias tróficas ou podem mover-se para águas superficiais ou subterrâneas (LARSEN; MOSEHOLM; NIELSEN, 1992). A constatação de contaminação do sedimento e plantas nessa área de estudo sinaliza a necessidade de promover ações de mitigação, bem como monitoramento, a fim de avaliar a eficácia das ações implementadas.

Em relação aos agrotóxicos, as análises revelaram que não há concentrações detectáveis das classes pesquisadas nas amostras de água, plantas e sedimento. Esses resultados podem ser explicados pela curta meia-vida de vários grupos de pesticidas, como os piretroides e organofosforados, ou ainda pela proibição dos organoclorados - que, apesar de possuírem grande período residual, foram banidos desde 1970 (Luvizotto-Santos et al., 2009), avaliando o uso de praguicidas nas atividades aquícolas inseridas na bacia hidrográfica do Rio Mogi-Guaçu, relataram que, nas entrevistas, $40 \%$ dos proprietários de pesqueiros e/ ou piscicultoras afirmaram que faziam uso de praguicidas durante as práticas de manejo, sendo diflubenzuron, paration metílico, triclorfon e triflumuron os mais utilizados. Todavia, não foram detectados 
resíduos desses praguicidas nas amostras de água, sedimentos e peixes coletadas tanto na baixa como na alta temporada de pesca, semelhantes aos achados desta pesquisa.

\section{CONCLUSÕES}

Conclui-se que após análises físico-químicas de temperatura, $\mathrm{pH}, \mathrm{OD}$, $\mathrm{DBO}$, fósforo total, alcalinidade, sólidos dissolvidos totais, fosfato, nitrato, nitrito, sulfato, amônia, fenol, metais pesados e agrotóxicos da água de uma piscicultura localizada às margens do Ribeirão da Mata, somente houve alteração da alcalinidade e sólidos totais, além de fenol e fosfato com valores acima dos limites permitidos para a classe II de água doce. Todavia, em 12 e 16 amostras de sedimento foram detectados chumbo e mercúrio, respectivamente, acima do VRQ.

\section{AGRADECIMENTOS}

Agradecemos à Fundação de Amparo à Pesquisa do Estado de Minas Gerais (FAPEMIG) e ao Conselho Nacional de Desenvolvimento Científico e Tecnológico $(\mathrm{CNPq})$ pelo apoio para a realização deste trabalho.

\section{REFERÊNCIAS}

ALLOWAY, B.J.; JACKSON, A.P.; MORGAN, H. (1990) The accumulation of cadmium by vegetables grown on soils contaminated from a variety of sources. Science of the Total Environment, v. 91, p. 223-236.

AMERICAN PUBLIC HEALTH ASSOCIATION (APHA). (2005) Standard Methods for the Examination Water and Wastewater. $21^{\text {st }}$ ed. Washington: APHA. 1083p.

AVILEZ, I.M.; DE AGUIAR, L.H.; HORI, T.S.; MORAES, G. (2013) Metabolic responses of matrinxã, Brycon amazonicus (Spix \& Agassiz, 1829), exposed to environmental nitrite. Aquaculture Research, v. 44, n. 4, p. 596-603.

BOLAÑOS, R.M.L.; VARESCHE, M.B.A.; ZAIAT, M.; FORESTI, E. (2OO1) Phenol degradation in horizontal-flow anaerobic immobilized biomass (haib) reactor under mesophilic conditions. Water Science and Technology, v. 44, n. 4, p. 167-174.

BOM, J.D. (2002) A influência da qualidade da água em reservatórios domiciliares na qualidade de vida da população de Umuarama - PR. 91 f. Dissertação (Mestrado em Engenharia de Produção) - Universidade Federal de Santa Catarina, Florianópolis.

CETESB - Companhia de Tecnologia de Saneamento Ambiental. (2005) Dispõe sobre a aprovação dos Valores Orientadores para Solos e Águas Subterrâneas no Estado de São Paulo - 2005, em substituição aos Valores Orientadores de 2001, e dá outras providências. Diário Oficial do Estado. Secretaria do Meio Ambiente, São Paulo, v. 115, n. 227, p. 22-23. Retificação 13.12.2005, v. 115, n. 233, p.42.

CONSELHO NACIONAL DO MEIO AMBIENTE - CONAMA. (2005) Resolução CONAMA no 357, de 17 de março de 2005. Dispõe sobre a classificação dos corpos de água e diretrizes ambientais para o seu enquadramento, bem como estabelece as condições e padrões de lançamento e efluentes, e dá outras providências. Diário Oficial da União, n.o 053, p. 58-63.

COSTA, O.F.T.; FERREIRA, D.J.S.; MENDONÇA, F.L.P.; FERNANDES, M.N. (2004) Susceptibility of the Amazonian fish, Colossoma macropomum (Serrasalminae) to short-term exposure to nitrite. Aquaculture, v. 232 p. 627-636
DAS, J.; PATRA, B.S.; BALIARSINGH, N.; PARIDA, K.M. (2006) Adsorption of phosphate by layered double hydroxides in aqueous solutions. Applied Clay Science, v. 32, p. 252-260.

DUBOROW, R.M.; CROSBY, D.M.; BRUNSON, M.W. (1997) Ammonia in fish ponds. Stoneville: Southern Regional Aquaculture Center (Publication 463).

EFSA - European Food Safety Authority. (2009) Cadmium in food scientific opinion of the Panel on Contaminants in the Food Chain The EFSA Journal, v. 980, p. 1-139.

GERMANO, P.M.L. \& GERMANO, M.I.S. (2001) Higiene e vigilância sanitária de alimentos. 3. ed. São Paulo: Varela. 629p.

HENDERSON, J.A.; BLOOD, D.C.; RADOSTITS, O.M. Clínica veterinária. 5. ed. Rio de Janeiro: Guanabara Koogan, 1983. p. 931-933.

IBGE - Instituto Brasileiro de Geografia e Estatística (portal do Governo Brasileiro). 2016. Disponível: http://cidades.ibge.gov.br/v3/ cidades/municipio/3149309. Acesso em: jan. 2017.

LARINI, L. (1987) Toxicologia. 2. ed. São Paulo: Manole. p. 114-120.

LARSEN, E.H.; MOSEHOLM, L.; NIELSEN, M.M. (1992) Atmospheric deposition of trace-elements around point sources and human health risk assessment. 2. Uptake of arsenic and chromium by vegetables grown near a wood preservation factory. Science of the Total Environment, v. 126, n. 3, p. 263-275.

LIN, Y.P. (2008) Simulating spatial distributions, variability and uncertainty of soil arsenic by geostatistical simulations in geographic information systems. Open Environmental Sciences, v. 2, p. 26-33.

LUKE, M.A., FROBERG, J.E., MASUMOTO, H.T. (1975) Extraction and cleanup of organochlorine, organophosphate, organonitrogen and hydrocarbon pesticides in produce for determination by gas-liquid chromatography. Journal of the Association of Official Analytical Chemists, v. 58, n. 5 , p. 1020-1026. 
LUVIZOTTO-SANTOS, R.; ÉLER, M.N.; ESPINDOLA, E.L.G.; VIEIRA, E.M. (2009) O uso de praguicidas nas pisciculturas e pesqueiros situados na bacia do rio Mogi-Guaçu. Boletim do Instituto de Pesca, v. 35, n. 3, p. 343-358.

MAIGA, A.; DIALLO, D.; BYE, R., PAULSEN, B.S. (2005) Determination of some toxic and essential metal ions in medicinal and edible plants from Mali. Journal of Agricultural and Food Chemistry, v. 53, n. 6, p. 2316-2321.

MULINARI, D.R.; SILVA, G.L.J.P.; RODRIGUES, L.A.; SILVA, M.L.C.P. (2007) Adsorção de íons fosfato nos compósitos celulose $/ \mathrm{ZrO}_{2}$. $\mathrm{nH}_{2} \mathrm{O}$ preparados pelos métodos da precipitação convencional e em solução homogênea. Cerâmica, v. 53, p. 345-353.

MURTA, P.H.G.; CEMBRANELLI, E.; LAICINI, Z.M.; MIGUEL, O. (1997) Estudos sobre o cádmio como contaminante de alimentos, com enfoque prioritário para laticínios. Higiene Alimentar, v. 11, n. 49, p. 14-17.

OGA, S.; CAMARGO, M.M.A.; BATISTUZZO, J.A.O. (2008) Fundamentos de toxicologia. 3. ed. São Paulo: Atheneu. 676p.

OSPAR COMMISSION - Protecting and conserving the North-East Atlantic and its Resources. (2002) Hazardous Substances Series: Cadmium. OSPAR Commission. 58p.

PACYNA, J.M. \& PACYNA, E.G. (2001) An assessment of global and regional emissions of trace metals to the atmosphere from anthropogenic sources worldwide. Environmental Review, v. 9, n. 4, p. 269-298.

PAOLIELLO, M.M.B. \& CHASIN, A.A.M. (2001) Ecotoxicologia do chumbo e seus compostos. Salvador: CRA. 144p.
PIETRO, M.B.; HIDALGO, A.; SERRA, J.L.; LLAMA, M.J. (2002) Degradation of phenol by Rhodococcus erythropolis UPV1 immobilized on Biolite ${ }^{\circledR}$ in a packed-bed reactor. Journal of Biotechnology, v. 97, n. 1, p. 1-11.

SORENSEN, E.M. (1991) Metal poisoning in fish. CRC Press, Boca Raton, Florida, Inc. 374p.

TAVARES, T.M. \& CARVALHO, F.M. (1992). Avaliação da exposição de populações humanas a metais pesados no ambiente: exemplos do Recôncavo Baiano. Química Nova, v. 15, p. 147-154.

TIŠLER, T. \& ZAGORC-KONČAN, J. (1997) Comparative assessment of toxicity of phenol, formaldehyde, and industrial wastewater to aquatic organism. Water Air Soil Pollution, v. 97, n. 3, p. 315-322.

ROSA, D.K. (2008) Análise custo/benefício da adoção de boas práticas de manejo em pesque-pague. 149 f. Dissertação (Mestrado) - Universidade Federal de São Carlos, São Carlos.

UNEP - United Nations Environment Programme. (2008) Draft final review of scientific information on cadmium.

USEPA - Agência de Proteção Ambiental dos Estados Unidos. (2001) Update of Ambient Water Quality Criteria for Cadmium.

YU-PIN, L.; BAI-YOUA, C.; GUEY-SHINB, S.; TSUN-KUOA, C. (2O10) Combining a finite mixture distribution model with indicator kriging to delineate and map the spatial patterns of soil heavy metal pollution in Chunghua County, central Taiwan. Environmental Pollution, v. 158, p. 235-244. 